Nature. 2010 January 7; 463(7277): 49-53. doi:10.1038/nature08637.

\title{
Preventing the return of fear in humans using reconsolidation update mechanisms
}

\author{
Daniela Schiller ${ }^{1,2}$, Marie-H. Monfils ${ }^{1,3}$, Candace M. Raio², David C. Johnson ${ }^{2}$, Joseph E. \\ LeDoux ${ }^{1}$, and Elizabeth A. Phelps ${ }^{1,2}$ \\ ${ }^{1}$ Center for Neural Science, New York University, New York, NY 10003, USA \\ ${ }^{2}$ Psychology Department, New York University, New York, NY 10003, USA \\ ${ }^{3}$ Psychology Department, University of Texas, Austin, TX 78712, USA
}

\section{Abstract}

Recent research on changing fears has examined targeting reconsolidation. During reconsolidation, stored information is rendered labile after being retrieved. Pharmacological manipulations at this stage result in an inability to retrieve the memories at later times, suggesting they are erased or persistently inhibited. Unfortunately, the use of these pharmacological manipulations in humans can be problematic. Here we introduce a non-invasive technique to target the reconsolidation of fear memories in humans. We provide evidence that old fear memories can be updated with non-fearful information provided during the reconsolidation window. As a consequence, fear responses were no longer expressed, an effect that lasted at least a year and was selective only to reactivated memories without affecting others. These findings demonstrate the adaptive role of reconsolidation as a window of opportunity to rewrite emotional memories, and suggest a non-invasive technique that can be used safely in humans to prevent the return of fear.

\begin{abstract}
Learning about potential dangers in the environment is critical for adaptive function, but at times fear learning can be maladaptive, resulting in excessive fear and anxiety. Research on changing fears has highlighted several techniques, most of which rely on the inhibition of the learned fear response. An inherent problem with these inhibition techniques is that the fear may return, for example with stress ${ }^{1}$. Recent research on changing fears targeting the reconsolidation process overcomes this challenge to some extent. During reconsolidation stored information is rendered labile after being retrieved, and pharmacological manipulations at this stage results in an inability to retrieve the memories at later times, suggesting they are either erased or persistently inhibited ${ }^{2-6}$. Although these pharmacological manipulations are potentially useful for changing learned fears, their use in humans can be problematic. Here we show that invasive techniques are not necessary to alter fear by targeting reconsolidation. This is based on the premise that reconsolidation is an adaptive update mechanism by which new information is incorporated into old memories ${ }^{3,7,8}$. By introducing new information during the reconsolidation period, it may be possible to permanently change the fear memory. In the present study, we provide evidence in humans that old fear memories can be updated with non-fearful information provided during the reconsolidation window. As a consequence, fear responses are no longer expressed. Furthermore, this effect is specific to the targeted fear memory and not others and
\end{abstract}


persists for at least a year. These findings demonstrate the adaptive role of reconsolidation as a window of opportunity to rewrite emotional memories, and suggest a non-invasive technique that can be used safely and flexibly in humans to prevent the return of fear.

\section{Pharmacological blockade of reconsolidation}

In contrast to the traditional view of memory formation as a one-time process of consolidation $^{9,10}$, the reconsolidation hypothesis suggests that memories are consolidated each time they are retrieved ${ }^{2-6}$. Evidence for reconsolidation of emotional memories comes from studies using pharmacological perturbation after retrieval ${ }^{11-13}$. The retrieval-induced plasticity allows the transition from a labile to a stable state after which memories are no longer prone to interference ${ }^{14}$. Why would such a recurrent window of vulnerability exist for old memories? From an evolutionary perspective, reconsolidation may serve as an adaptive update mechanism allowing for new information, available at the time of retrieval, to be integrated into the initial memory representation ${ }^{3,7,8}$. This view captures the fluidity of memory and suggests a dynamic process through which emotional memories are formed, updated and maintained.

Using Pavlovian fear conditioning as a model paradigm, research in non-human animals has detailed the molecular processes involved in emotional memory reconsolidation by pharmacologically blocking various stages of this process, after which the memory was no longer expressed. Most of these studies utilize protein synthesis inhibitors, or other pharmacological agents, that are not safe for use in humans $3,4,6,11-14$. Because the ability to impair emotional memories has important implications for the treatment for anxiety disorders linked to traumatic memories, such as post-traumatic stress disorder (PTSD), identifying techniques to target reconsolidation that can be used flexibly and safely in humans is critical. One possibility is to capitalize on reconsolidation as an update mechanism. If an old fear memory could be re-stored while incorporating neutral or more positive information provided at the time of retrieval, it may be possible to permanently modify the fearful properties of this memory.

Although this approach captures the very essence of reconsolidation, it has been surprisingly neglected in emotion research in humans and other animals. To date, there is only one demonstration of this approach in non-human animals using fear conditioning ${ }^{8}$, and efforts to alter fear memories by introducing non-fearful information during initial consolidation have had mixed results ${ }^{15-17}$. In humans, studies of motor and declarative memory suggest new information presented during the reconsolidation window may interfere with the older memories by either impairing the memory ${ }^{18}$ or modifying it to incorporate the new information ${ }^{7,19}$. However, there is robust evidence that motor, declarative and emotional memories rely on distinct memory systems in the brain ${ }^{20}$, and the reconsolidation process and impact of new information presented during the reconsolidation window may differ depending on the type of memory updated.

\section{Interference of reconsolidation using extinction}

In the present study, we sought to capitalize on reconsolidation as an update mechanism and attempted to alter emotional memories with new information. We hypothesized that updating a fear memory with non-fearful information, provided through extinction training, would rewrite the original fear response and prevent the return of fear. A recent study in rats $^{8}$, provides strong evidence in support of this hypothesis. Briefly, $24 \mathrm{hr}$ following fear conditioning, rats were reminded of the conditioned stimulus (CS), using a single retrieval trial, and subsequently underwent extinction training. The extinction phase was conducted either within or outside the reconsolidation window, which lasts about 6 hours $^{11,18}$. It was found that fear responses returned only in rats that underwent extinction after 
reconsolidation was completed. In contrast, rats that had extinction training during the reconsolidation window did not show recovery of fear.

To test this hypothesis in humans, we designed two experiments examining whether extinction training conducted during the reconsolidation window would block the return of extinguished fear. In the first study, three groups of subjects underwent fear conditioning using a discrimination paradigm with partial reinforcement (Fig. 1a). Two colored squares were used. One square (CS+) was paired with a mild shock to the wrist (unconditioned stimulus, US) on 38\% of the trials whereas the other square was never paired (CS-). A day later, all three groups underwent extinction training where the two CSs were repeatedly presented without the US. In two groups the fear memory was reactivated prior to extinction using a single presentation of the CS+. One group $(n=20)$ received the reminder trial 10 min before extinction (within the reconsolidation window), whereas the second group ( $n=$ 23) was reminded 6 hours prior to extinction (outside the reconsolidation window ${ }^{11,18}$ ). The third group $(n=22)$ was not reminded of the fear memory before extinction training. Twenty-four hours later, all three groups were presented again with the CSs without the aversive outcome (re-extinction) in order to assess spontaneous fear recovery. The measure of fear was skin conductance response (SCR). At each stage, the differential fear response was calculated by subtracting responses to the $\mathrm{CS}-$ from responses to the $\mathrm{CS}+$.

The results of the spontaneous recovery experiment are presented in Figure 1b (see also supplementary Fig. S1). Subjects that showed successful levels of fear acquisition and extinction were included in the analysis. We verified that these levels were equivalent between the groups using two-way ANOVAs with main effects of group (10 min, $6 \mathrm{hr}$, and no-reminder) and time (early and late phase). For both acquisition and extinction there was a significant main effect of time $\left(F_{1,62}=9.92, p<0.05 ; F_{1,62}=19.59, p<0.01\right.$, respectively $)$ but no effect of group or interaction. Follow up $t$-tests confirmed that subjects had significantly stronger responses to CS+ compared to CS- during acquisition (late phase; 10 min group: $t=2.68, p<0.05$; $6 \mathrm{hr}$ group: $t=3.72, P<0.05$; no-reminder group; $t=3.72, P$ $<0.05$ ), but by the last trial of extinction there was no difference (10 min group: $t=-0.94$, $\mathrm{NS}$; $6 \mathrm{hr}$ group: $t=-0.23$, NS; no-reminder group; $t=-0.79$, NS).

The decrease in fear responses from acquisition (late phase) to extinction (last trial) for each group was assessed with a two-way ANOVA with main effects of group (10 min, $6 \mathrm{hr}$, and no-reminder) and time (acquisition, extinction). This revealed a significant main effect of time $\left(F_{1,62}=29.9, P<0.01\right)$, but no effect of group or interaction. Follow up $t$-tests confirmed the reduction of fear in all three groups (10 min group: $t=2.70, P<0.05 ; 6 \mathrm{hr}$ group: $t=4.06, P<0.05$; no-reminder group; $t=4.07, P<0.05$ ), and there was no difference in the level of fear reduction between the groups $(P>0.5$ for all three comparisons).

Spontaneous recovery was assessed using a two-way ANOVA with main effects of group (10 $\mathrm{min}, 6 \mathrm{hr}$, and no-reminder) and time (early and late phase of re-extinction, defined by the mean first 4 responses versus the subsequent 4 , respectively) revealing a significant main effect of time $\left(F_{1,62}=6.26, P<0.05\right)$, and a group $\times$ time interaction $\left(F_{2,62}=4.63, P<\right.$ $0.05)$. Follow up $t$-tests compared the differential responses between the last trial of extinction and the first trial of re-extinction. Spontaneous recovery was found in subjects who did not receive a reactivation trial prior to extinction $(t=2.69, P<0.05)$, or underwent extinction 6 hours after fear reactivation $(t=2.66, P<0.05)$. In contrast, subjects that had extinction $10 \mathrm{~min}$ after reactivation showed no spontaneous recovery $(t=0.28, \mathrm{NS})$. These results suggest that the spontaneous recovery of fear following extinction can be prevented if extinction training is conducted during the time window in which the fear memory is hypothesized to be undergoing reconsolidation. 


\section{Persistence of reconsolidation blockade}

In this initial study, we used a $24 \mathrm{hr}$ interval to test for long-term memory, which, for practical reasons, is the standard in human fear recovery experiments ${ }^{25-27}$. However, if the fear memory is persistently altered, as would be predicted if we are impacting reconsolidation of the fear memory, we would expect this effect to last for much longer time intervals. In an attempt to examine if the observed blockade of fear memory persists, we invited the participants for a follow-up test after approximately a year (10-14 months). Nineteen of the 65 original participants were located and included in the follow-up study (10 min group, $n=8$; $6 \mathrm{hr}$ group, $n=4$; no-reminder group, $n=7$ ). We collapsed subjects from the two groups previously showing spontaneous recovery (i.e., $6 \mathrm{hr}$ and no-reminder) into one group. As mentioned above, following the spontaneous recovery test, subjects were reextinguished using 10 non-reinforced presentations of the stimuli ensuring that all subjects showed no evidence of conditioned fear at the conclusion of the initial experiment. This reextinction allowed us to conduct a second test of fear recovery a year later. For this second recovery test, we used a more potent recovery essay, namely reinstatement, in which subjects were exposed to 4 unsignaled shocks, followed by non-reinforced presentations of the CS. The index of fear recovery (Fig. 2 and supplementary Fig. S2) was the difference in the conditioned fear response at the end of re-extinction following the initial spontaneous recovery test and the conditioned fear response immediately after reinstatement a year later. Specifically, a differential SCR score (CS+ minus CS-) was calculated for the end of reextinction and post-reinstatement. A two-way ANOVA with main factors of group (10 min, $6 \mathrm{hr} /$ no-reminder) and stage (re-extinction, post-reinstatement) showed a significant main effect of group $\left(F_{1,17}=5.89, P<0.05\right)$. The group $\times$ stage interaction was marginally significant $\left(F_{1,17}=2.78, P<0.07\right.$, one-tail). Follow up one-tail $t$-test comparisons revealed that reinstatement was significant in the $6 \mathrm{hr} /$ no-reminder group $(t=2.12, P<0.03)$, but not the 10 min group $(t=0.22$, NS). Moreover, the magnitude of reinstatement (that is, the difference in conditioned fear at the end of re-extinction and following reinstatement a year later) was significantly larger in the $6 \mathrm{hr} /$ no-reminder group than the $10 \min$ group $(t=$ $1.75, p<0.05)$. Lastly, a comparison of post-reinstatement conditioned fear between the groups showed a significant difference $(t=2.18, P<0.03)$.

These results suggest that reactivation of a fear memory renders it labile and extinction training during this lability period leads to a long lasting blockade of recovery of fear. In contrast, recovery of fear a year later was observed after regular extinction training. Fear recovery was also observed when extinction training was conducted after a sufficient temporal gap following reactivation, presumably allowing for reconsolidation to be complete.

\section{Specificity of reconsolidation blockade}

If interfering with reconsolidation using extinction is to be clinically useful, it is also important to determine whether it is specific. In real life situations, a traumatic event can be associated with multiple cues, and each could potentially trigger the recollection of the event and elicit fear reactions. In order to assess the specificity of this fear blockade technique, we examined whether interfering with the reconsolidation of one fear predictive cue would affect the fate of another, associated, cue.

In a second experiment, more than one stimulus was associated with the same aversive outcome (Fig. 3a). Specifically, using a within-subject design, subjects underwent fear conditioning using three colored squares. Two squares $(\mathrm{CS} a+$ and $\mathrm{CS} b+)$ were paired with the shock on $38 \%$ of the trials. The third square (CS-) was never paired with the shock. A day later, subjects received a single presentation of CSa+ and the CS-, but not CS $b+$. Ten 
minutes following the reminder trial, extinction training was conducted (within the reconsolidation window) using repeated presentations of all CSs without the aversive outcome. Reinstatement of the fear memory was conducted $24 \mathrm{hr}$ later, when subjects returned to the experiment room and received 4 unsignalled presentations of the shock. Ten minutes later the CSs were presented without the aversive outcome (re-extinction).

The results of the experiment are presented in Figure 3b (see also supplementary Fig. S3). Subjects $(n=18)$ that showed successful fear acquisition and extinction were included. We verified that these levels were equivalent between the two conditioned stimuli (CSa+ and $\mathrm{CS} b+$ ) using two-way ANOVAs with main effects of stimulus (CSa+, CS $b+$ and CS-) and time (early and late phase, defined by the mean response during the first and second half of each phase, respectively). In acquisition, there was a significant main effect of stimulus $\left(F_{2,51}=3.51, P<0.05\right)$ and a stimulus $\times$ time interaction $\left(F_{2,51}=3.27, P<0.05\right)$. In extinction, there was a significant main effect of time $\left(F_{1,51}=48.74, P<0.01\right)$. Follow up $t$ tests were used to further assess acquisition and extinction of fear. We compared the mean SCR to CSa+ or CS b+ with the CS- during the second half of the acquisition session. Subjects showed significantly stronger responses to CSa+ compared to CS- $(t=6.01, P<$ $0.05)$, as well as to CS $b+$ compared to CS- $(t=6.68, P<0.05)$. Moreover, the level of acquisition to $\mathrm{CS} a+$ and $\mathrm{CS} b+$ was equivalent $(t=0.76$, NS). To assess fear extinction, we compared the mean SCR to CSa+ or CS $b+$ with the CS- during the last trial of extinction. There were no significant differences in responses to CSa+ compared to CS- $(t=-0.26$, $\mathrm{NS}$ ), or to CS $b+$ compared to CS $-(t=-0.56$, NS), and responses to CS $a+$ and $\mathrm{CS} b+$ were equally extinguished $(t=0.23$, NS). Moreover, subjects had successful reduction of fear, as assessed by comparing the SCR during the second half of acquisition with the last trial of extinction, to both $\mathrm{CS} a+(t=2.62, P<0.05)$ and $\mathrm{CS} b+(t=4.08, P<0.05)$ but not to the CS $-(t=-0.09, \mathrm{NS})$, which was low to begin with.

To assess the recovery of fear, we used a two-way ANOVA with main effects of stimulus $(\mathrm{CS} a+, \mathrm{CS} b+$ and CS-) and time (early and late phase of re-extinction, defined by the mean first 4 responses versus the last 4 , respectively), which revealed a stimulus $\times$ time interaction $\left(F_{2,51}=5.14, P<0.01\right)$. Using follow up $t$-tests, we compared the SCR during the last trial of extinction (before reinstatement) with the first trial of re-extinction (following reinstatement). Subjects showed reinstated fear responses only to CS $b+$, which is the stimulus that was not reminded prior to extinction $(t=2.16, P<0.05)$. In contrast, fear responses to $\mathrm{CS} a+$, which was reminded $10 \mathrm{~min}$ before extinction training, did not recover $(t$ $=0.22$, NS $)$. As expected, there were also no fear responses to the CS- $(t=0.16 \mathrm{NS})$. Thus, extinction during reconsolidation affected only the reactivated memory and no other trace associated with the original event.

\section{Discussion}

The present findings suggest a novel technique to target specific fear memories and prevent the return of fear following extinction training. Using two recovery assays, we demonstrated that extinction conducted during the reconsolidation window of an old fear memory prevented the spontaneous recovery or the reinstatement of fear responses, an effect that was maintained a year later. Moreover, this manipulation selectively affected only the reactivated CS while leaving fear memory to other non-reactivated CS intact.

It has been suggested that the adaptive function of reconsolidation is to allow old memories to be updated each time they are retrieved ${ }^{3,7,8}$. In other words, our memory reflects our last retrieval of it rather than an exact account of the original event. This notion has received support from interference paradigms targeting motor and declarative memories ${ }^{7,18,19}$. These studies demonstrate that new information provided during reconsolidation could affect old 
memories by modifying or interfering with them, but in contrast to the present study, they do not provide evidence for memory blockade. This difference in the impact of new information presented during reconsolidation on the subsequent qualities of different types of memory may be due to the diverse nature of the underlying memory systems. For instance, unlike the distributed cortical representation of declarative memories ${ }^{20}$, conditioned fear has a more discrete neural representation localized in the amygdala ${ }^{21}$. Indeed, in the lateral amygdala pharmacological blockade of the molecular cascade engaged by retrieval prevents the reconsolidation of fear memories in rats ${ }^{4}$. This raises the possibility that our behavioral manipulation, namely, extinction training during reconsolidation, targeted the same molecular mechanism.

Although the current behavioral study does not provide direct evidence that a process of reconsolidation mediates the effects of extinction training, support for this hypothesis comes from recent findings in rats ${ }^{8}$. Following fear consolidation, a single isolated retrieval trial prior to extinction prevented the recovery of fear in rats. Interestingly, plasticity in the lateral amygdala induced by the CS retrieval was impaired by the presentation of a CS one hour later, indicating possible interference with the reconsolidation process, similar to the interference caused to reconsolidation by pharmacological blockade in rats ${ }^{4}$. Together, these findings reveal cross-species similarities, which may reflect an evolutionarily preserved adaptive mechanism whereby the neural representation of fear memory can be significantly altered through timing dependent molecular mechanisms triggered by exposure to feareliciting stimuli.

The current results also suggest that timing may play a more important role in the control of fear than previously appreciated. Standard extinction training, without prior memory reactivation, also triggers the fear memory. Given this, one might expect mere extinction training to have similar effects. That is, the first trial of extinction might serve as the reminder cue triggering the reconsolidation cascade, which is immediately followed by extinction. However, there is abundant evidence that during standard extinction training the non-reinforced presentations of the fear-eliciting cue induce new inhibitory learning, which competes for expression with the initial fear learning resulting in the recovery of fear responses in some circumstances ${ }^{17,23-27}$. Our findings suggest that the timing of extinction relative to the reactivation of the memory can capitalize on reconsolidation mechanisms. Two factors may be important determinants in this process: the timing of extinction training relative to retrieval, and/or the chunking of the CS presentations during extinction relative to reactivation (that is, the fact that they are massed relative to the single retrieval trial during the reconsolidation phase). Further studies are required to disentangle these possibilities.

In conclusion, the present study showed that updating fear memories with non-fearful information provided through extinction training led to the blockade of previously learned fear responses and a lasting change in the original fear memory. These results have significant implications for the treatment of anxiety disorders. Current forms of therapy rely heavily on extinction ${ }^{28-29}$, but the fact that extinguished fear could recover under certain conditions dampens the resilience of anxiety patients following treatment. The discovery that certain pharmacological manipulation can potentially erase memories through effects on reconsolidation has been encouraging; however, most compounds showing such effects in various species are toxic to humans. Recently, there has been promising evidence using compounds that are testable on humans, namely beta-adrenergic receptor blockers ${ }^{30}$, which also show effects in trauma patients ${ }^{31}$, but these effects are not observed every case ${ }^{32}$. The present study proposes that such invasive techniques are not necessary. Using a more natural intervention that captures the adaptive purpose of reconsolidation allows a safe and easily implemented way to prevent the return of fear. 


\section{Methods (For online)}

\section{Experiment 1}

The paradigm consisted of three consecutive stages conducted $24 \mathrm{hr}$ apart: Day 1 Acquisition, Day 2 - Reactivation and Extinction, and Day 3 - Re-extinction (Fig. 1a). During acquisition, three randomly assigned groups of subjects underwent a Pavlovian discrimination fear conditioning paradigm with partial reinforcement. The CSs were yellow and blue squares $(4 \mathrm{sec})$ and the US was a mild shock to the wrist $(200 \mathrm{msec})$ co-terminating with the CS+. The CS+ was paired with the shock on a $38 \%$ partial reinforcement schedule and the CS- was never paired with shock. Subjects were instructed to pay attention to the computer screen and try to figure out the relationship between the stimuli appearing on the screen and the shocks. A day later, all three groups underwent extinction training where the CS+ and CS- were repeatedly presented without the US. In two groups, the fear memory was reactivated prior to extinction. During reactivation, the CS+ was presented once (unreinforced), followed by a 10 min break. One group $(n=20)$ underwent extinction after the 10 min break (within the reconsolidation window). The second group $(n=23)$ underwent extinction $6 \mathrm{hr}$ after the reactivation (outside of the reconsolidation window). In the third group $(n=22)$, the fear memory was not reactivated. Following the break, extinction immediately followed for half the subjects in this group, or was conducted $6 \mathrm{hr}$ later for the other half. During the break, all participants watched a pre-selected TV show episode. Day 3 consisted of re-extinction where participants were presented with nonreinforced presentations of the stimuli. During all sessions (acquisition, reminder, extinction and reextinction), with the exception of the breaks, the participants were attached to the SCR and shock electrodes, and the shock stimulator was set to the 'On' position.

To examine how long the blockade of memory persists, we invited the participants of the experiment to come back to the lab after about a year (10-14 months). Twenty three participants were located (10 min group, $n=10 ; 6 \mathrm{hr}$ group, $n=5$; no-reminder group, $n=$ 8). As mentioned above, following the spontaneous recovery test, subjects were reextinguished using 10 non-reinforced presentations of the stimuli, which allowed us to reassess their recovery of fear. We used a more potent recovery essay, namely, reinstatement, in which subjects were exposed to 4 unsignaled shocks, followed by nonreinforced presentations of the same CSs that were used in the spontaneous recovery experiment (10 presentations each, using two randomized orders counterbalanced across subjects). The index of fear recovery was the difference in the conditioned fear response at the end of re-extinction following the initial spontaneous recovery test and the conditioned fear response immediately after reinstatement a year later. Specifically, a differential SCR score (CS+ minus CS-) was calculated for the end of re-extinction (mean of last 2 trials) and post-reinstatement (mean of first 4 trials). We collapsed subjects from the two groups previously showing spontaneous recovery (i.e., $6 \mathrm{hr}$ and no-reminder) into one group. Subjects that failed to re-extinguish following the spontaneous recovery test (differential SCR score > 0.2) or showed no measurable responses to the shocks during reinstatement were not included in the analysis ( 4 subjects). The final analysis included 19 subjects (10 min group, $n=8 ; 6 \mathrm{hr} /$ no-reminder group, $n=11$ ). Throughout the session, the participants were attached to the SCR and shock electrodes, and the shock stimulator was set to the 'On' position.

\section{Experiment 2}

The paradigm consisted of three consecutive stages conducted $24 \mathrm{hr}$ apart: Day 1 Acquisition, Day 2 - Reactivation and Extinction, and Day 3 - Reinstatement and Reextinction, using a within-subject design (Fig. 2a). During Acquisition, subjects underwent fear conditioning using three colored squares. Two squares (CSa+ and CS $b+$ ) were paired 
with the shock on a 38\% partial reinforcement schedule. The third square (CS-) was never paired with the shock. Subjects were instructed to pay attention to the computer screen and try to figure out the relationship between the stimuli appearing on the screen and the shocks. Day 2 consisted of reactivation and extinction. During reactivation, the CSa+ and the CSwere each presented once (unreinforced), in a counterbalanced fashion. Participants were then given a $10 \mathrm{~min}$ break where they watched a pre-selected TV show episode. Extinction immediately followed and consisted of nonreinforced presentations of the three stimuli (CSa $+, \mathrm{CS} b+, \mathrm{CS}-)$. Day 3 consisted of reinstatement and re-extinction. During reinstatement, subjects were administered with 4 unsignalled shocks. After a 10 min break, a re-extinction session commenced where participants were presented with nonreinforced presentations of the three stimuli (CSa+, CS $b+, \mathrm{CS}-$ ). During all sessions, (acquisition, reminder, extinction, reinstatement and reextinction), with the exception of the breaks, the participants were attached to the SCR and shock electrodes, and the shock stimulator was set to the 'On' position.

\section{Psychophysiological stimulation and assessment}

Mild shocks were delivered through a stimulating bar electrode attached with a Velcro strap to the right inner wrist. A Grass Medical Instruments stimulator (West Warwick, Rhode Island) charged by a stabilized current was used. Subjects determined the level of the shock themselves, beginning at a very mild level of shock $(10 \mathrm{~V})$ and gradually increasing the level until the shock reached the maximum level that they determined was uncomfortable, but not painful (the maximum level was $60 \mathrm{~V}$ ). All shocks were given for $200 \mathrm{~ms}$, with a current of 50 pulses per second.

Skin conductance response (SCR) was assessed using two $\mathrm{Ag}-\mathrm{AgCl}$ electrodes, which were connected to a BioPac Systems (Santa Barbara, California) skin conductance module. The electrodes were attached to the first and second fingers of the left hand, between the first and second phalanges. SCR waveforms were analyzed offline, using AcqKnowledge 3.9 software (BIOPAC Systems Inc., Goleta, California). SCR amplitudes to the CS and US were the dependent measures of conditioned and unconditioned responses, respectively. The level of SCR response was determined by taking the base to peak difference for the first waveform (in micro siemens, $\mu \mathrm{s}$ ) in the 0.5 to $4.5 \mathrm{~s}$ window following stimulus onset. The minimal response criterion was $0.02 \mu \mathrm{s}$. The raw SCR scores were square root transformed to normalize distributions. These normalized scores were scaled according to each subject's unconditioned response by dividing each response by the mean square-root-transformed US response.

\section{Supplementary Material}

Refer to Web version on PubMed Central for supplementary material.

\section{Acknowledgments}

The authors thank Keith Doelling for assistance with data collection and discussions on the revised version of the manuscript. We also thank Yael Niv and Mohammed Milad for advice on the experimental protocols. This study was funded by James S. McDonnell Foundation and NIH R21 MH072279 grants (E.A.P.), NIH grants R37 MH038774, P50 MH058911, RO1 MH046516, and K05 MH067048 (J.E.L.), Postdoctoral fellowships NSERC, CIHR, and AHFMR (M.-H.M.), and a Fulbright award (D.S.).

\section{References}

1. Miracle AD, Brace MF, Huyck KD, Singler SA, Wellman CL. Chronic stress impairs recall of extinction of conditioned fear. Neurobio. Learn. Mem. 2006; 85:213-218. 
2. Misanin JR, Miller RR, Lewis DJ. Retrograde amnesia produced by electro-convulsive shock after reactivation of a consolidated memory trace. Science. 1968; 160:203-204. [PubMed: 4230606]

3. Alberini CM. Mechanisms of memory stabilization: are consolidation and reconsolidation similar or distinct processes? Trends Neuro. 2005; 28:51-56.

4. Nader K, Schafe GE, LeDoux JE. Fear memories require protein synthesis in the amygdala for reconsolidation after retrieval. Nature. 2000; 406:722-726. [PubMed: 10963596]

5. Dudai Y. Reconsolidation: the advantage of being refocused. Curr. Opin. Neurobiol. 2006; 16:174178. [PubMed: 16563730]

6. Sara SJ, Hars B. In memory of consolidation. Learn. Mem. 2006; 13:515-521. [PubMed: 17015848]

7. Hupbach A, Gomez L, Hardt O, Nadel R. Reconsolidation of episodic memories: A subtle reminder triggers integration of new information. Learn. Mem. 2007; 14:47-53. [PubMed: 17202429]

8. Monfils M-H, Cowansage KK, Klann E, LeDoux JE. Extinction-reconsolidation boundaries: Key to persistent attenuation of fear memories. Science. 2009; 324:951-955. [PubMed: 19342552]

9. Squire LH, Davis HP. The pharmacology of memory: a neurobiological perspective. Annu. Rev. Pharmacol.Toxicol. 1981; 21:323-356. [PubMed: 7016009]

10. McGaugh JL. Memory-a century of consolidation. Science. 2000; 287:248-251. [PubMed: 10634773]

11. Duvarci S, Nader K. Characterization of fear memory reconsolidation. J. Neurosci. 2004; 24:9269_ 9275. [PubMed: 15496662]

12. Alberini CM, Milekic MH, Tronel S. Memory: Mechanisms of memory stabilization and destabilization. Cell Mol Life Sci. 2006; 63:999-1008. [PubMed: 16596332]

13. Lee JL, Milton AL, Everitt BJ. Reconsolidation and extinction of conditioned fear: Inhibition and potentiation. J. neurosci. 2006; 26:10051-10056. [PubMed: 17005868]

14. Doyere V, Debiec J, Monfils MH, Schafe GE, LeDoux JE. Synapse-specific reconsolidation of distinct fear memories in the lateral amygdala. Nat. neurosci. 2007; 10:414-416. [PubMed: 17351634]

15. Myers KM, Ressler KJ, Davis M. Different mechanisms of fear extinction dependent on length of time since fear acquisition. Learn. Mem. 2006; 13:216-223. [PubMed: 16585797]

16. Alvarez RP, Johnson L, Grillon C. Contextual-specificity of short-delay extinction in humans: Renewal of fear-potentiated startle in a virtual environment. Learn. Mem. 2007; 14:247-253. [PubMed: 17412963]

17. Schiller D, Cain CK, et al. Evidence for recovery of fear following immediate extinction in rats and humans. Learn. Mem. 2008; 15:394-402. [PubMed: 18509113]

18. Walker MP, Brakefield T, Hobson JA, Stickgold R. Dissociable stages of human memory consolidation and reconsolidation. Nature. 2003; 425:616-620. [PubMed: 14534587]

19. Forcato, et al. Reconsolidation of declarative memory in humans. Learn. Mem. 2007; 14:295-303. [PubMed: 17522018]

20. Squire, LH.; Knowlton, BJ. The New Cognitive Neurosciences. Gazzaniga, MS., editor. Cambridge: MIT Press; 2000. p. 765-780.

21. Ledoux JE. Emotion circuits in the brain. Ann. Rev. Neurosci. 2000; 23:155-184. [PubMed: 10845062]

22. Lee JL, Everitt BJ, Thomas KL. Independent cellular processes for hippocampal memory consolidation and reconsolidation. Science. 2004; 304:839-843. [PubMed: 15073322]

23. Bouton ME. Context, ambiguity, and unlearning : sources of relapse after behavioral extinction. Bio. Psychiatry. 2002; 52:976-986. [PubMed: 12437938]

24. Quirk GJ, Mueller D. Neural mechanisms of extinction learning and retrieval. Neuropsychopharmacology. 2008; 33:56-72. [PubMed: 17882236]

25. Phelps EA, Delgado MR, Nearing KI, LeDoux JE. Extinction learning in humans: role of the amygdala and vmPFC. Neuron. 2004; 43:897-905. [PubMed: 15363399]

26. Kalisch, et al. Context-dependent human extinction memory is mediated by a ventromedial prefrontal and hippocampal network. J. Neurosci. 2006; 26:9503-11. [PubMed: 16971534]

27. Milad, et al. Recall of fear extinction in humans activates the ventromedial prefrontal cortex and hippocampus in concert. Bio. Psychiatry. 2007; 62:446-454. [PubMed: 17217927] 
28. Foa EB, Franklin ME, Moser J. Context in the clinic : how well do cognitive-behavioral therapies and medications work in combination. Biol. Psychiatry. 2002; 52:987-997. [PubMed: 12437939]

29. Rauch SL, Shin LM, Phelps EA. Neurocircuitry models of posttraumatic stress disorder and extinction: human neuroimaging research—past, present and future. Biol. Psychiatry. 2006; 60:376-382. [PubMed: 16919525]

30. Kindt M, Soeter M, Vervliet B. Beyond extinction: erasing human fear responses and preventing the return of fear. Nat. Neurosci. 2009; 12:256-58. [PubMed: 19219038]

31. Brunet A, et al. Effect of post-retrieval propranolol on psychophysiologic responding during subsequent script-driven traumatic imagery in post-traumatic stress disorder. J. Psychiatr. Res. 2008; 42:503-506. [PubMed: 17588604]

32. Tollenaar MS, Elzinga BM, Spinhoven P, Everaerd W. Psychophysiological responding to emotional memories in healthy young men after cortisol and propranolol adminitration. Psychopharmacology. 2009; 203:793-803. [PubMed: 19139852] 
a

Day 1 Day 2 Day 3

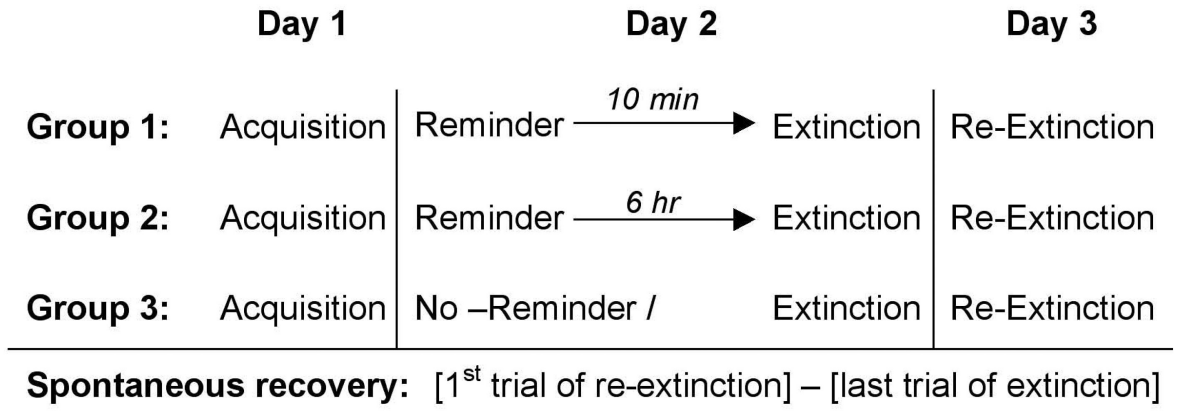

b

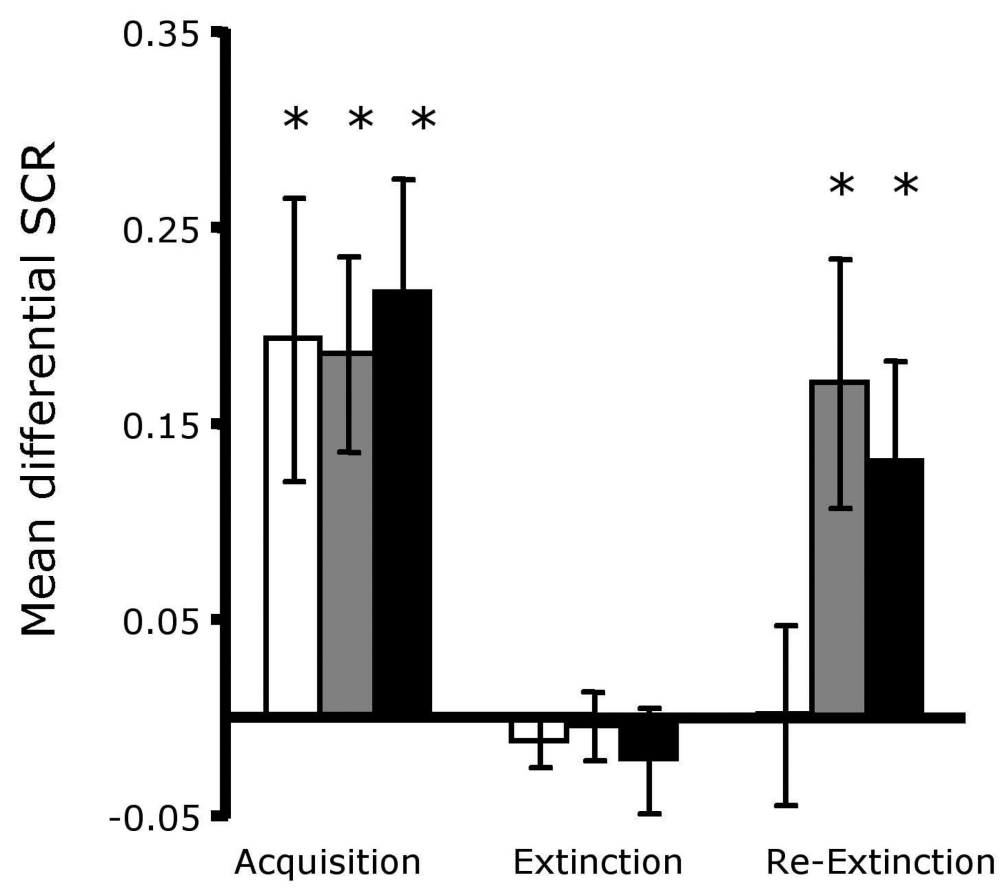

Figure 1. Extinction during reconsolidation prevents spontaneous recovery of extinguished fear (a) Experimental design and timeline. (b) Mean differential SCRs (CS+ minus CS-) during acquisition (late phase), extinction (last trial) and re-extinction (first trial) for each experimental group (10 min reminder, $6 \mathrm{hr}$ reminder, and no-reminder). The three groups showed equivalent fear acquisition and extinction. Spontaneous recovery (first trial of reextinction versus the last trial of extinction) was found in the group that had not been reminded or that was reminded 6 hours prior to extinction. In contrast, there was no spontaneous recovery in the group reminded 10 min before extinction. *Significant difference between acquisition and extinction, or extinction and re-extinction within group at $p<0.05$; Error bars represent standard errors 


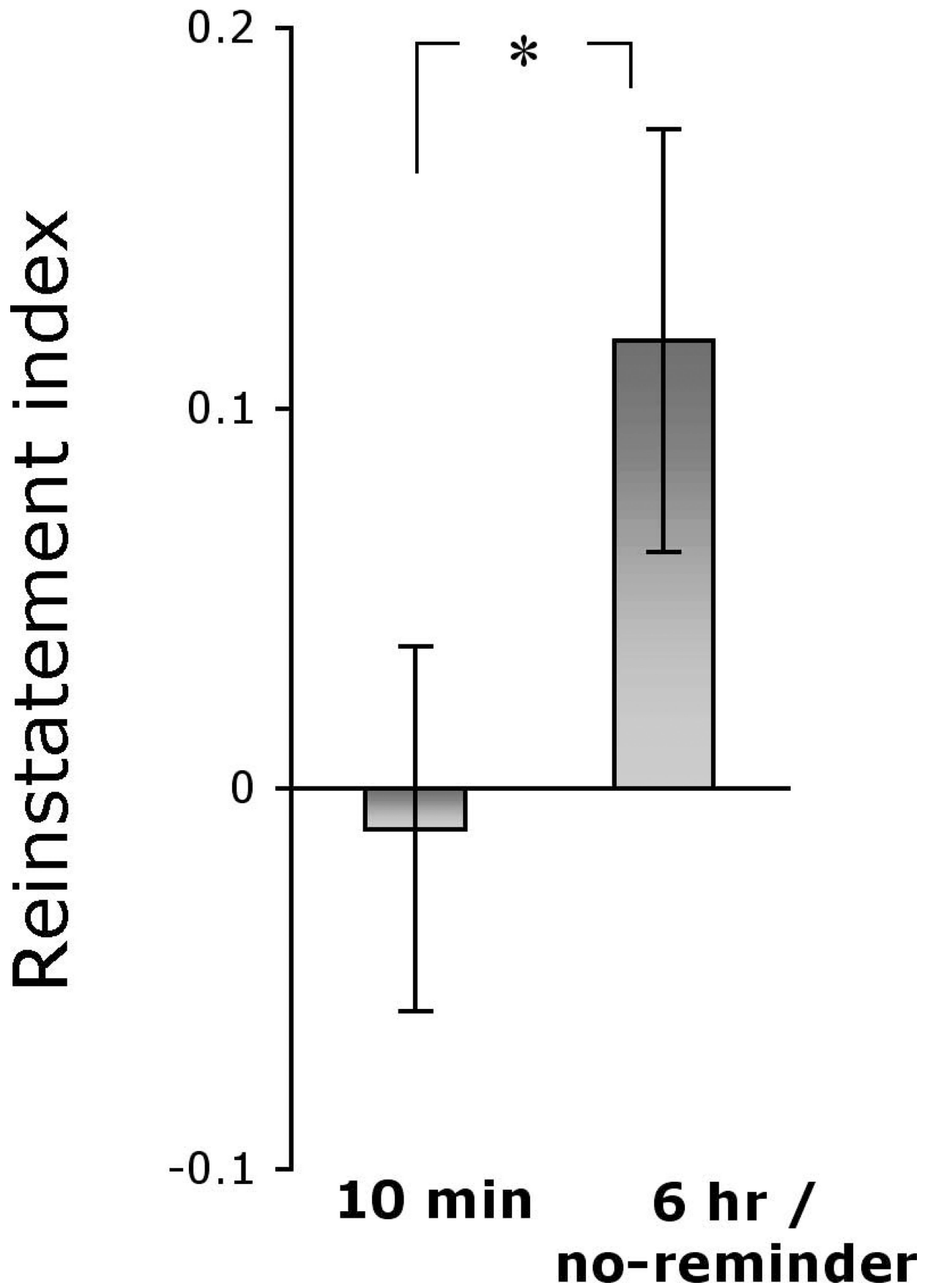

Figure 2. Blockade of the return of fear persists one year later

The reinstatement index is the difference in the conditioned fear response (CS+ minus $\mathrm{CS}-$ ) at the end of re-extinction following the initial spontaneous recovery test and the conditioned fear response immediately after reinstatement a year later. The magnitude of the reinstatement was significantly higher in the $6 \mathrm{hr} /$ no-reminder group compared to the 10 min group, which showed no reinstatement. *Significant difference at $p<0.05$; Error bars represent standard errors 
a

Day 2

Day 3

\begin{tabular}{c|c|c}
\hline Acquisition & Reminder & Reinstatement \\
$\mathrm{CS} a+\mathrm{CS} b+\mathrm{CS}-$ & $\mathrm{CS} a+\mathrm{CS}-$ & $4 \times \mathrm{US}$ \\
& $10 \mathrm{~min}$ & $10 \mathrm{~min}$ \\
& $\begin{array}{c}\text { Extinction } \\
\mathrm{CS} a+\mathrm{CS} b+\mathrm{CS}-\end{array}$ & $\begin{array}{c}\text { Re-Extinction } \\
\mathrm{CS} a+\mathrm{CS} b+\mathrm{CS}-\end{array}$ \\
\hline
\end{tabular}

Reinstatement: $\left[1^{\text {st }}\right.$ trial of re-extinction] - [Last trial of extinction]

b
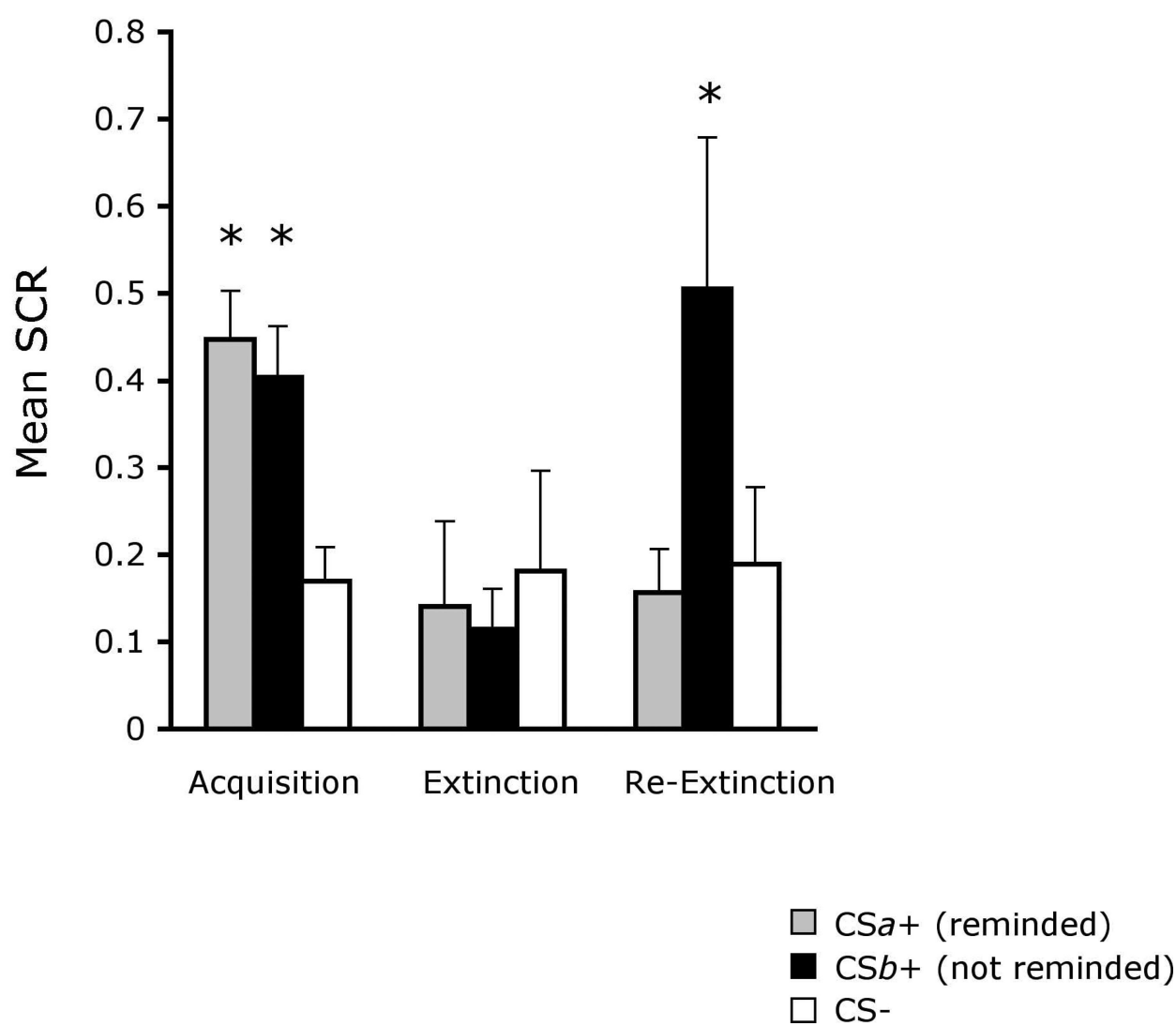

Figure 3. Extinction during reconsolidation prevents reinstatement of extinguished fear (a) Experimental design and timeline. (b) Mean SCRs (CS $a+, \mathrm{CS} b+, \mathrm{CS}-$ ) during acquisition (late phase), extinction (last trial) and re-extinction (first trial). Subjects had equivalent levels of acquisition and extinction of conditioned fear to the two conditioned stimuli. The index of fear recovery was the first trial of re-extinction (following reinstatement) minus the last trial of extinction (before reinstatement). Fear reinstatement was found only to $\mathrm{CS} b+$, (not reminded before extinction training) but not $\mathrm{CS} a+$ (reminded 10 min before extinction training). *Significant difference between acquisition and 
extinction, or extinction and re-extinction for each stimulus at $p<0.05$; Error bars represent standard errors 